\title{
Tunable optical and dielectric properties of polymeric composite materials based on magneso-silicate
}

\author{
M M ABOU-MESALAM ${ }^{1}$, M R ABASS ${ }^{1}$, A B IBRAHIM ${ }^{1}$, A M ELSEMAN ${ }^{2,3, *}$ (iD and A M HASSAN ${ }^{4}$ \\ ${ }^{1}$ Atomic Energy Authority, Hot Labs Centre, Cairo 13759, Egypt \\ ${ }^{2}$ Advanced Materials Division, Electronic and Magnetic Materials Department, Central Metallurgical Research and \\ Development Institute (CMRDI), Helwan, Cairo 11421, Egypt \\ ${ }^{3}$ State Key Laboratory of Alternate Electrical Power System with Renewable Energy Sources, North China Electric Power \\ University, Beijing 102206, People's Republic of China \\ ${ }^{4}$ Chemistry Department, Faculty of Science, Al-Azhar University, Nasr City, Cairo, Egypt \\ *Author for correspondence (AhmedMourtada5555@yahoo.com; amourtada@ncepu.edu.cn)
}

MS received 18 September 2017; accepted 13 April 2018; published online 2 February 2019

\begin{abstract}
Magneso-silicate (MgSi) as an inorganic ion exchange material was synthesized by a precipitation technique. Then, the MgSi was impregnated into polyacrylamide acrylic acid and its composites (Poly) by condensation polymerization. To study the effect of $\gamma$-radiation, the polyacrylamide acrylic acid and its $\mathrm{MgSi}$ samples were synthesized using $\gamma$-irradiating systems at 25, 65 and $90 \mathrm{kGy}$. The variations in the radiation dose and amorphous structure were altered and confirmed by $\mathrm{X}$-ray diffraction (XRD). Moreover, the absorbance and band-gap energy were enhanced by inserting MgSi into the polymeric composites (Poly). Furthermore, variations in temperature with dielectric constant, dielectric loss and conductivity of the samples at various frequencies from 100, 500, 1000, 2000 to $4000 \mathrm{~Hz}$ have been explained.
\end{abstract}

Keywords. Magneso-silicate; polymeric composites; dielectric properties; radiation.

\section{Introduction}

In recent years, polymers have been developed in different fields such as transport, electronic and pharmaceutical industries because of their notable material properties [1,2]. Nowadays, polymers are attracting great interest because of their low cost, low weight, easy processability, high-quality surface, easy fabrication of thick and thin samples, etc. One of the most valuable advantages of polymers is their capacity to inhibit electrical conductivity as insulators, but now they are attracting considerable attention as conducting polymers $[3,4]$. In addition, useful polymers with controlled refractivity, photochromic, electrochromic and optoelectronic capacities have been produced recently [5-9]. Furthermore, the use of polymers with tunable refractive properties as optical modulators, optical filters or electro-optic waveguide devices have also been reported [10-12].

On the other hand irradiation of polymers has also emerged as a novel way of modifying the polymer properties. Irradiation of polymers alters the structure by cross connecting, free-radical development, irreversible bond cleavages and so forth which results in the discontinuity of atoms and arrangement of immersed and unsaturated groups $[13,14]$. All these procedures induce defects inside the material that affect its optical, electrical, and mechanical and conduction properties. Changes in optical properties are significant as they can be used to create optical sensors and light emitting diodes (LEDs) [15-17].

Recently, much work has been done on polymer composites. Starting from the continuous improvement towards the scaling down of electronics to high dielectric steady polymer composites, they have turned out to be promising materials for installed capacitor applications [18-20]. On the other hand, the polymers having low dielectric constants in the scope of 2-5 generally utilized as a part of low leakage capacitors are adaptable, easy to fabricate and result in high dielectric constant [21-23].

In this work, magneso-silicate $(\mathrm{MgSi})$ as an inorganic ion exchange material was synthesized using a precipitation technique. Then, the polyacrylamide acrylic acid and its $\mathrm{MgSi}$ composites were synthesized using $\gamma$-radiation for initiating polymerization at radiation doses of 25,65 and $90 \mathrm{kGy}$. The prepared composite materials were investigated using different analytical techniques and presented a new ion-exchange characteristic compared to the original ones.

\section{Experimental}

\subsection{Materials}

Acrylamide (AM) $\left(\mathrm{C}_{3} \mathrm{H}_{5} \mathrm{NO} ; \mathrm{BDH}, \mathrm{UK}\right)$, acrylic acid (AA) $\left(\mathrm{C}_{3} \mathrm{H}_{4} \mathrm{O}_{2}\right.$; Merck, Germany), acetone $\left(\left(\mathrm{CH}_{3}\right)_{2} \mathrm{CO}\right.$; Aldrich 


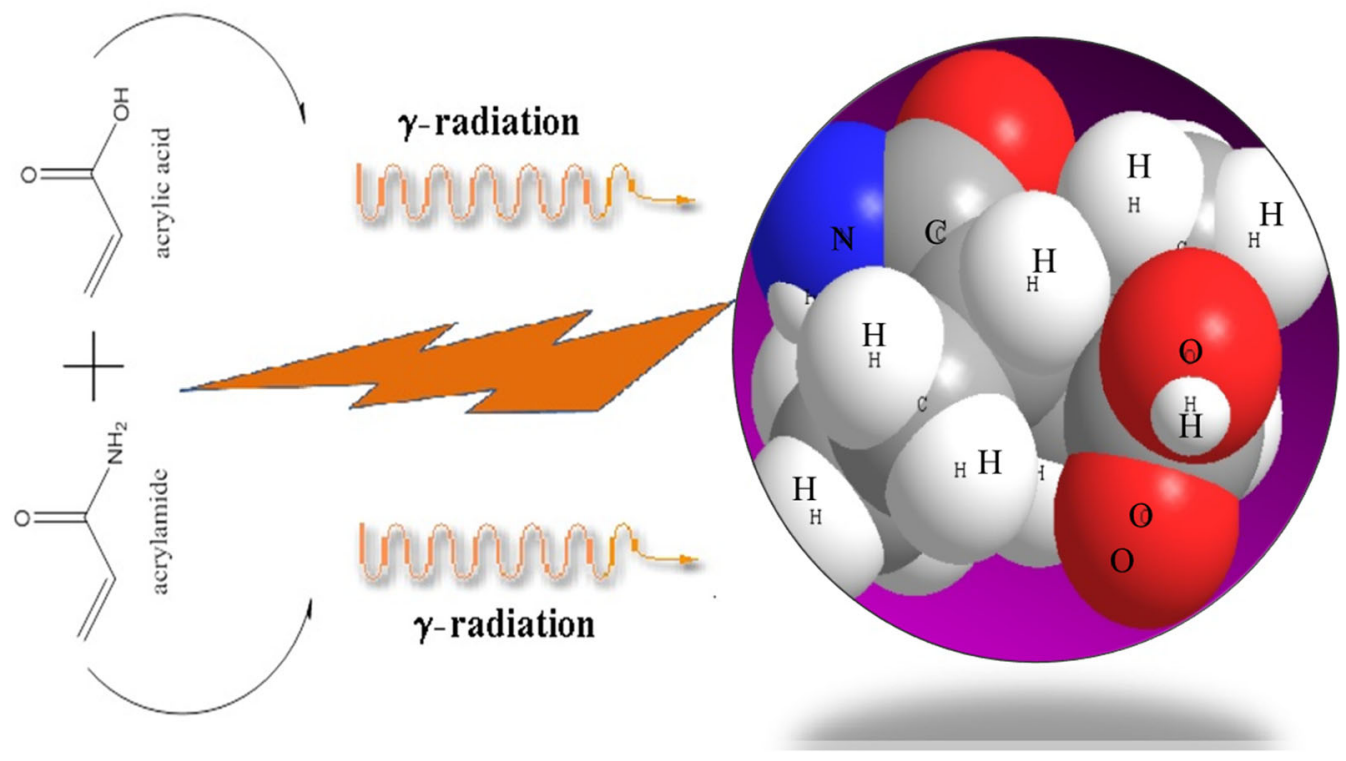

Figure 1. The possible copolymerization reaction of AM with AA by irradiation to give a molecular structure of cross-linking acrylamide AA copolymers (Poly).

products, Germany), magnesium(II) chloride hexahydrate $\left(\mathrm{MgCl}_{2} \cdot 6 \mathrm{H}_{2} \mathrm{O} ;\right.$ ADWIC, Egypt) and sodium metasilicate $\left(\mathrm{Na}_{2} \mathrm{SiO}_{3}\right.$; Avondale, England) were used. All reagents and chemicals used were of analytical grade and used without further purification.

\subsection{Synthesis of co-monomer solutions (Poly)}

The investigated monomer solutions of AM and AA are prepared by dissolving $10 \%$ of each monomer in deoxygenated water. Then, the AM monomer solution is mixed dropwise with aqueous solutions of AA under constant stirring at room temperature. Polyacrylamide AA copolymers (Poly) are prepared as shown in figure 1. The co-monomer solutions were irradiated under different doses of 25, 65 and $90 \mathrm{kGy}$ with a dose rate of $1.05 \mathrm{kGy} \mathrm{h}^{-1}$ to obtain Poly 25 , Poly 65 and Poly 90, respectively.

\subsection{Synthesis of $M g S i$}

MgSi was prepared by dropwise addition of equimolar solutions $(0.5 \mathrm{M})$ of $\mathrm{Na}_{2} \mathrm{SiO}_{3}$ to $\mathrm{MgCl}_{2} \cdot 6 \mathrm{H}_{2} \mathrm{O}$ with a volumetric ratio of $\mathrm{Mg} / \mathrm{Si}=1: 5$ under constant stirring at room temperature. After the addition was complete, diluted ammonia solution was added until a precipitate was obtained and then the reaction mixture was kept undisturbed overnight. The precipitate was washed with $0.1 \mathrm{M} \mathrm{HNO}_{3}$ to remove $\mathrm{Cl}^{-}$ions and impurities. Then, the precipitate was washed with dilute water to remove $\mathrm{NO}_{3}^{-}$ions. It was filtered and washed several times with deionized water. The precipitate was dried at $50^{\circ} \mathrm{C}$ and sieved for different mesh sizes and stored at room temperature.

\subsection{Synthesis of polymeric composite materials}

Polyacrylamide AA MgSi (MgSi Poly) was synthesized by dropwise addition of Poly to equimolar solutions $(0.5 \mathrm{M})$ of $\mathrm{MgSi}$ under constant stirring at room temperature. Then, MgSi Poly 25, MgSi Poly 65 and MgSi Poly 90 composites were prepared by subjecting $\mathrm{MgSi}$ Poly to $\gamma$-radiation at doses 25,65 and $90 \mathrm{kGy}$ with a dose rate of $1.05 \mathrm{kGy} \mathrm{h}^{-1}$, respectively. After irradiation, the obtained hydrogel was cut into small pieces with stainless-steel scissors, soaked in acetone for removal of unreacted monomers, washed with water, dried at $50^{\circ} \mathrm{C}$, grained and sieved for different mesh sizes.

\subsection{Physical characterization and measurements}

$\mathrm{X}$-ray diffraction (XRD) patterns of the resulting products were recorded by using a Bruker D8-advanced X-ray powder diffractometer with $\mathrm{Cu} \mathrm{K} \alpha$ radiation $(\lambda=1.5406 \AA)$. The micrographs of the produced samples were inspected by direct observation via a scanning electron microscope (SEM) (model JSM-5400, JEOL Instrument, Japan). The elemental composition was determined by energy-dispersive X-ray (EDX) analysis. IR spectra were obtained by mixing the solid with $\mathrm{KOH}$ in a ratio of 1:5 and ground to a very fine powder. A transparent disc was formed under a moisturefree atmosphere. IR spectra were recorded using a BOMEM FTIR spectrometer in the range of $400-4000 \mathrm{~cm}^{-1}$. UV-Vis absorption and diffuse reflectance spectra were recorded at room temperature using a UV-VIS-NIR spectrophotometer (Jasco-V-570 spectrophotometer, Japan) fitted with an integrating sphere reflectance unit (ISN) in the wavelength range of 200-2000 nm. The dielectric and conductivity properties 
were measured using a network impedance analyser (HIOKI 3532-50 LCR Hi TESTER) in the frequency range of $1 \mathrm{~Hz}-5 \mathrm{kHz}$. The artificial sources of current to produce an electrical potential field were grounded. An electrometer and two probe heads were used for the characterization of the samples.

\section{Results and discussion}

In this paper, the $\gamma$-radiation was utilized for the crosslinking of the AM and AA hydrogels. The scheme of polymerization reaction of $\mathrm{AM}$ with $\mathrm{AA}$ by $\gamma$-beam radiation is shown in figure 1. It can be seen that a mixture of AM with AA was irradiated; $a$ bond in the twofold obligation of AM with AA was broken and monomer radicals were shaped. These monomer radicals were consolidated together and the AM/AA copolymer was achieved.

\subsection{Crystal structure results}

The XRD patterns of Poly copolymers at radiation doses of 25,65 and $90 \mathrm{kGy}$ are shown in figure $2 \mathrm{a}$. From this figure, it can be seen that Poly composites have an amorphous structure and a broad peak in the range of $15^{\circ}$ to $30^{\circ}$ [24,25]. There is no doubt that the crystallinity of the synthetic samples was affected by the radiation dose. It increased with increasing radiation doses as revealed in figure $2 \mathrm{a}$. However, the sample which was irradiated with $90 \mathrm{kGy}$ has a crystalline structure compared to other samples. These results were similar to the data obtained from the XRD of polyacrylamide-co-acrylic acid prepared by Hassan et al $[24,26]$. The peaks at $2 \theta 13.8^{\circ}$ and $16.8^{\circ}$ correspond to the (100) and (020) crystalline peaks of Poly 90. This confirms the partial crystallization of Poly 90 units in the copolymer and the semi-crystalline structure [27].

Also, the addition of MgSi to the radiated copolymer added some features to the new materials. Accordingly, figure $2 b$ reveals the XRD patterns of MgSi Poly 25, MgSi Poly 65 and MgSi Poly 90 composites which have been prepared at radiation doses of 25, 65 and $90 \mathrm{kGy}$, respectively. It can be found that the different indexed peaks at 30.5, 31.1, 45.3, 55.5 and $75.70^{\circ}$ correspond to (031), (131), (231), (321) and (360) in all the MgSi Poly composite patterns (XRD JCPDS data file no. 43-1022) [28,29], indicating that it is enriched with crystalline moieties. The reason for this is attributed to the presence of MgSi composites in the prepared sample. Other peaks appearing at 13.8 and $16.8^{\circ}$ are attributed to the presence of acrylamide AA copolymers [30]. Noticeably, the crystalline characteristics of MgSi Poly samples increased with radiation dose from 25 to $90 \mathrm{kGy}$. This could explain the nature and similar behaviour of the prepared samples at radiation doses of 90 and $65 \mathrm{kGy}$ which are crystalline in nature. The increase of crystallinity of Poly 90 and $\mathrm{MgSi}$ Poly 90 in the final stage of irradiation might be due to molecular disintegration and rearrangement within the acrylamide AA copolymers by which copolymer gets exposed and
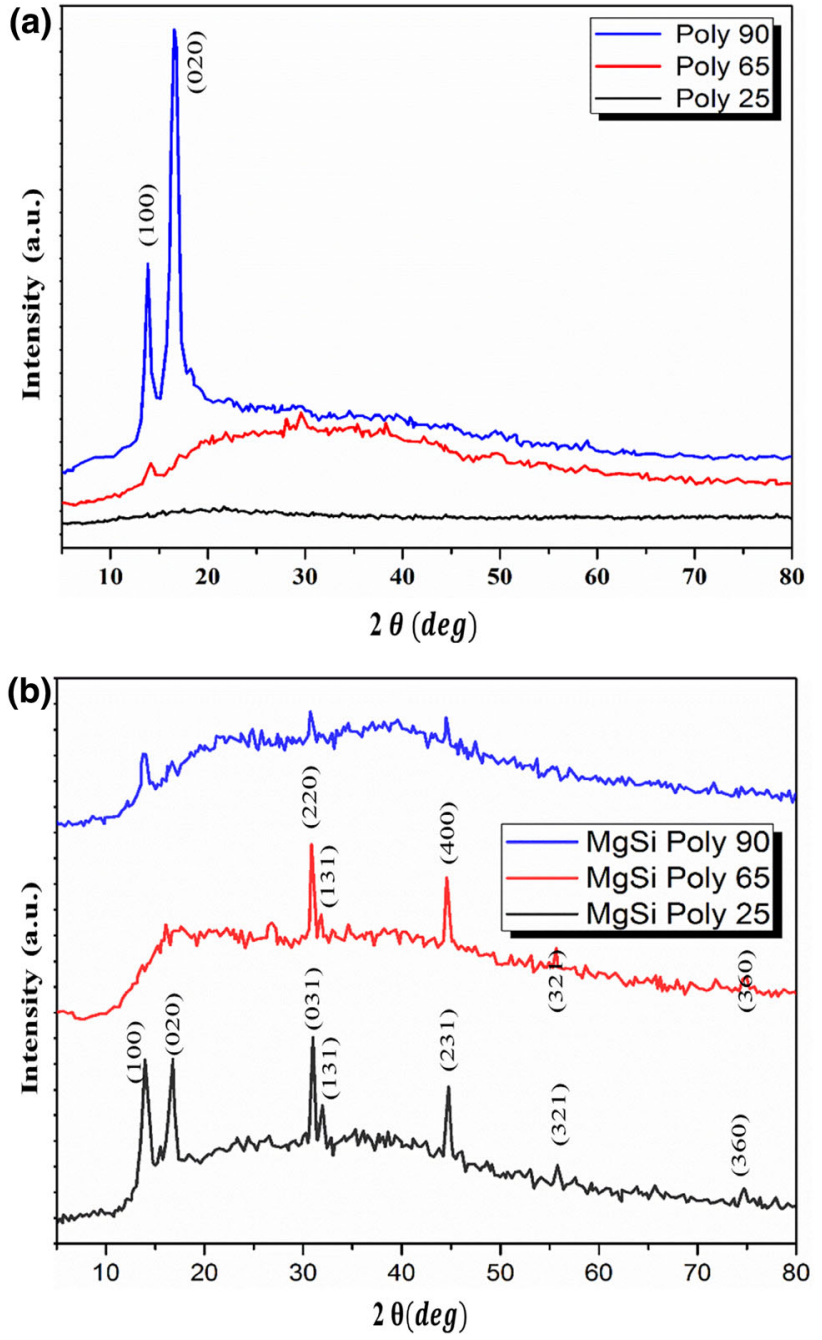

Figure 2. Powder XRD patterns for (a) Poly 25, 65 and 90 and (b) MgSi Poly 25, 65 and 90 samples.

converted to the amorphous state with prolonged irradiation. The decrease in the crystallinity index is mainly due to the conversion of the crystalline form of acrylamide AA copolymers to the amorphous form.

\subsection{Morphology structures}

Figure 3 shows the SEM images of all Poly and MgSi Poly composite films. All samples were examined in solid shape as shown in figure $3 \mathrm{a}-\mathrm{f}$. It can be seen that the SEM micrographs, in figure $3 a$ and $b$, appeared wrinkled for the Poly 25 and Poly 65 samples. Figure $3 \mathrm{c}$ shows that the surface form of the specimen seems to have a compact morphology with a coarse surface distribution. On the other hand in figure $3 \mathrm{~d}$ and e, the surface morphology of MgSi Poly 25 and MgSi Poly 65 films appeared uneven and harsh, and some cubic states of various sizes were evident in a specific area. Whereas, figure $3 \mathrm{f}$ shows the SEM image of the highest radiation of MgSi Poly 90. 

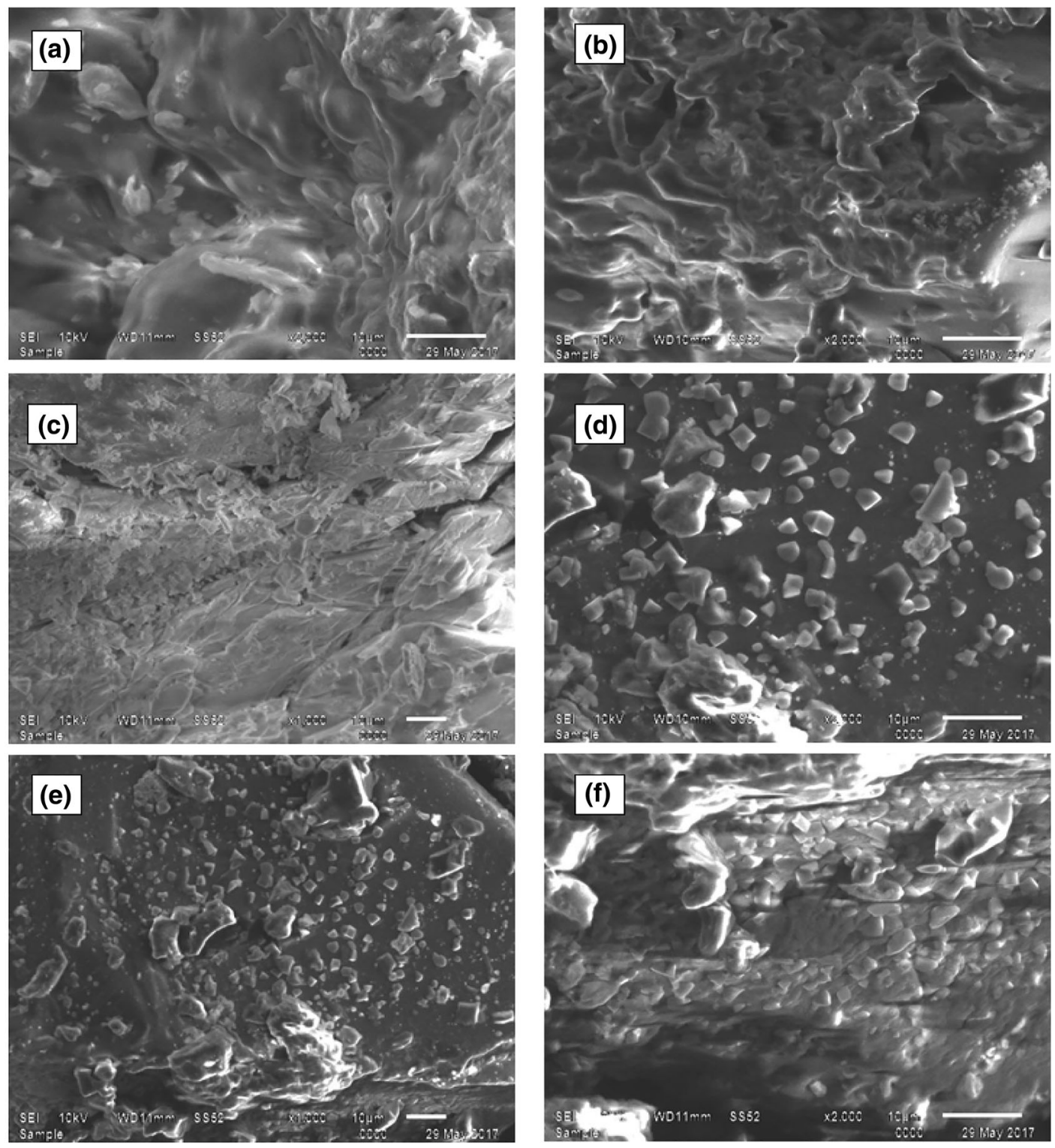

(g)

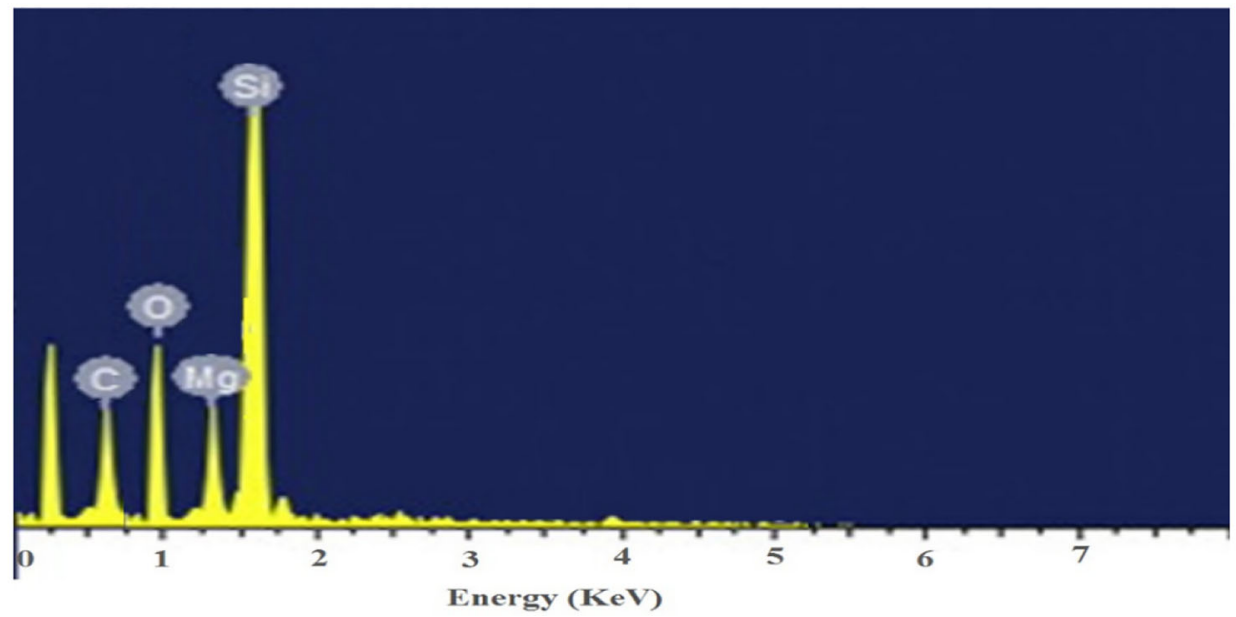

Figure 3. SEM micrographs of (a) Poly 25, (b) Poly 65, (c) Poly 90, (d) MgSi Poly 25, (e) MgSi Poly 65, (f) MgSi Poly 90 samples and (g) EDX analysis of MgSi Poly 25. 
The morphology appears blended with small and vast wrinkles connected together with a couple of cubic shapes. Finally, in figure $3 \mathrm{~g}$ the selected EDX micrograph of $\mathrm{MgSi}$ Poly 25 shows clearly distinguishable phases of $\mathrm{Mg}, \mathrm{Si}, \mathrm{O}$ and $\mathrm{C}$, and the resulting relative atomic percentages of different metals at the points are: $\mathrm{Si}=45.2 \%, \mathrm{Mg}=20.3 \%, \mathrm{O}=$ $15.1 \%, \mathrm{C}=11.6 \%$ and $\mathrm{N}=7.7 \%$.
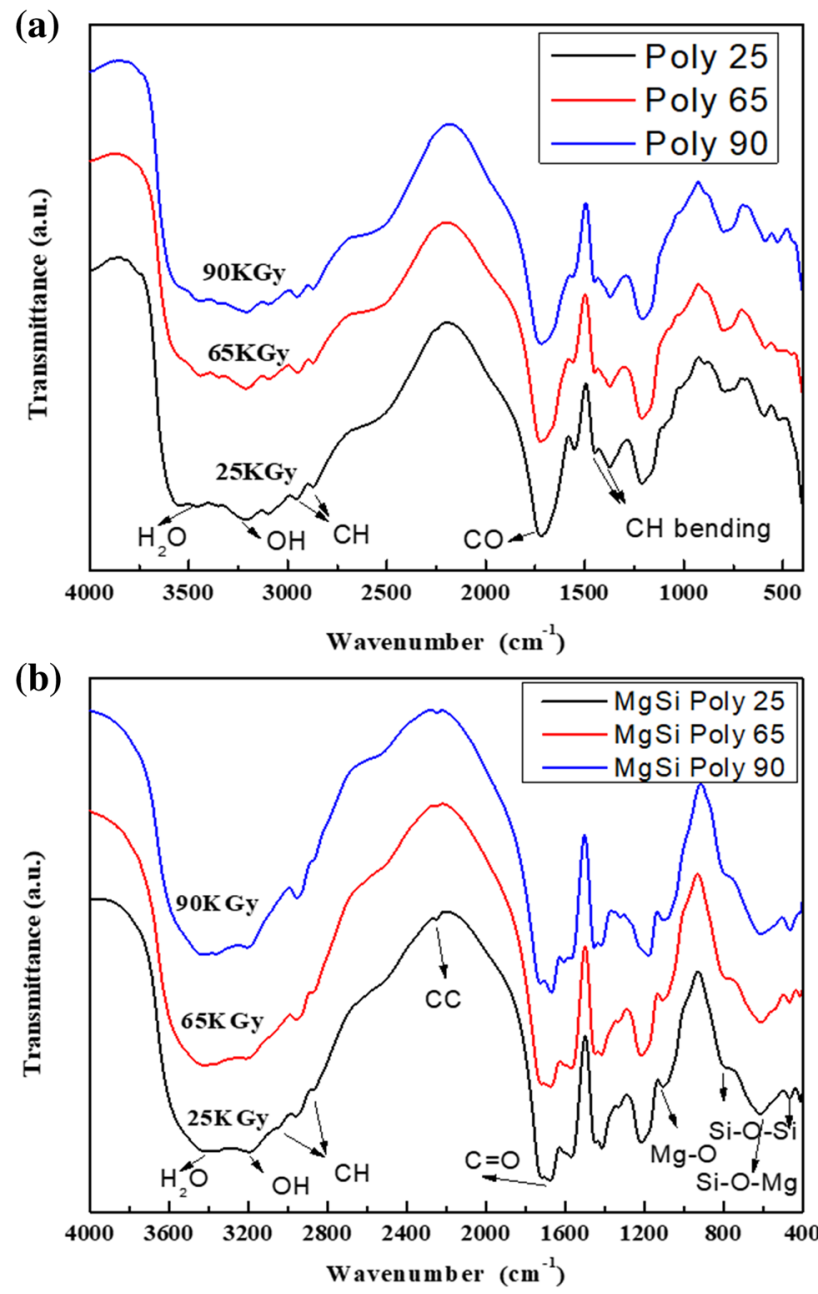

Figure 4. FT-IR spectra of (a) Poly 25, Poly 65 and Poly 90 and (b) MgSi Poly 25, MgSi Poly 65 and MgSi Poly 90 samples.

\subsection{Fourier transform infrared spectra}

The IR spectra of Poly copolymers prepared at radiation doses of 25, 65 and $90 \mathrm{kGy}$ are presented in figure 4a and table 1 . The spectrum of Poly copolymers displays a broadened band at $3255-3500 \mathrm{~cm}^{-1}$ due to the stretching vibration both of the hydroxyl groups and water molecules [31-33]. While, the bands appearing at 3090, 2950 and $2870 \mathrm{~cm}^{-1}$ may be due to the stretching mode of $\mathrm{C}-\mathrm{H}$ of AM and AA [34]. The strong band appearing at $1717-1731 \mathrm{~cm}^{-1}$ is attributed to the stretching mode of $\mathrm{C}=\mathrm{O}$ of $\mathrm{AA}$ [34]. A strong band appearing at $1549-1563 \mathrm{~cm}^{-1}$ is attributed to the bending mode of the $\mathrm{N}-\mathrm{H}$ bond of AM [35]. Moreover, two bands appearing at 1450 and $1370 \mathrm{~cm}^{-1}$ may be attributed to the bending mode of $\mathrm{C}-\mathrm{H}$ of AM and AA. Two broad bands appeared at 1213 and $1022 \mathrm{~cm}^{-1}$ and are attributed to the bending vibration of $\mathrm{C}-\mathrm{N}$ of AM [36]. On the other hand, the IR spectra of $\mathrm{MgSi}$ Poly composites prepared at radiation doses of 25, 65 and $90 \mathrm{kGy}$ are presented in figure $4 \mathrm{~b}$. This figure shows that a broad band observed at $3460-3200 \mathrm{~cm}^{-1}$ can be attributed to the stretching mode of water and $\mathrm{OH}$ groups absorbed on the composites [37]. The bands appearing at 2960-2875 $\mathrm{cm}^{-1}$ may be due to the stretching mode of $\mathrm{C}-\mathrm{H}$ of $\mathrm{AM}$ and AA. A weak band appeared at $2245 \mathrm{~cm}^{-1}$ due to the stretching mode of the $\mathrm{C} \equiv \mathrm{C}$ bond and may be present by rearrangement in the structure [38]. The bands appearing at 1675-1663 $\mathrm{cm}^{-1}$ are due to the stretching mode of the $\mathrm{C}=\mathrm{O}$ group of AA [34]. The band appearing at $1571-1561 \mathrm{~cm}^{-1}$ is due to the bending mode of the N-H bond of AM. Two bands appeared at 1315$1413 \mathrm{~cm}^{-1}$ and may be attributed to the bending mode of $\mathrm{C}-\mathrm{H}$ of AM and AA. A band appeared at $1104 \mathrm{~cm}^{-1}$, this band reflects the metal oxygen bond $\mathrm{Mg}-\mathrm{O}$ [39]. Three bands appeared at 793, 611 and $465 \mathrm{~cm}^{-1}$. The first band may be attributed to $\mathrm{Mg}-\mathrm{OH}$ deformation vibration or overlapping of the $\mathrm{Si}-\mathrm{O}$ and $\mathrm{Si}-\mathrm{OH}$ and $\mathrm{Mg}-\mathrm{O}$ bonds in the structure $[39,40]$. The second band may be attributed to the $\mathrm{Si}-\mathrm{O}-\mathrm{Mg}$ bending vibrations $[39,40]$. The third band may be due to the $\mathrm{Si}-\mathrm{O}-\mathrm{Si}$ bending vibrations, the bands at 1104, 793, 611 and $465 \mathrm{~cm}^{-1}$ indicating the impregnation of $\mathrm{Mg}$ and $\mathrm{Si}$ into the polymeric resin.

\subsection{Optical properties}

Photoactive materials were extensively characterized as the materials react to light or electromagnetic radiation [41].

Table 1. FT-IR spectral data of Poly 25, 65 and 90, MgSi Poly 25, 65 and 90 samples.

\begin{tabular}{lccccccccc}
\hline Sample & $\nu_{\mathrm{H}_{2} \mathrm{O}}$ & $v_{\mathrm{O}-\mathrm{H}}$ & $v_{\mathrm{C}-\mathrm{H}}$ stretching & $v_{\mathrm{C}=\mathrm{O}}$ & $\nu_{\mathrm{N}-\mathrm{H}}$ bending & $v_{\mathrm{C}-\mathrm{H}}$ bending & $v_{\mathrm{M}-\mathrm{O}}$ & $v_{\mathrm{Si}-\mathrm{O}-\mathrm{Mg}}$ & $\nu_{\mathrm{Si}-\mathrm{O}-\mathrm{Si}}$ \\
\hline Poly 25 & 3482 & 3222 & $3090-2863$ & 1717 & 1549 & $1374-1451$ & - & - & - \\
Poly 65 & 3454 & 3243 & $3088-2870$ & 1725 & 1563 & $1366-1444$ & - & - & - \\
Poly 90 & 3464 & 3234 & $3087-2871$ & 1731 & 1562 & $1367-1451$ & - & - & - \\
MgSi Poly 25 & 3433 & 3208 & $2960-2875$ & 1675 & 1570 & $1310-1413$ & 1106 & 614 & 467 \\
MgSi Poly 65 & 3447 & 3209 & $2961-2872$ & 1663 & 1563 & $1315-1415$ & 1099 & 613 & 474 \\
MgSi Poly 90 & 3440 & 3209 & $2963-2874$ & 1665 & 1561 & $1316-1420$ & 1105 & 611 & 460 \\
\hline
\end{tabular}




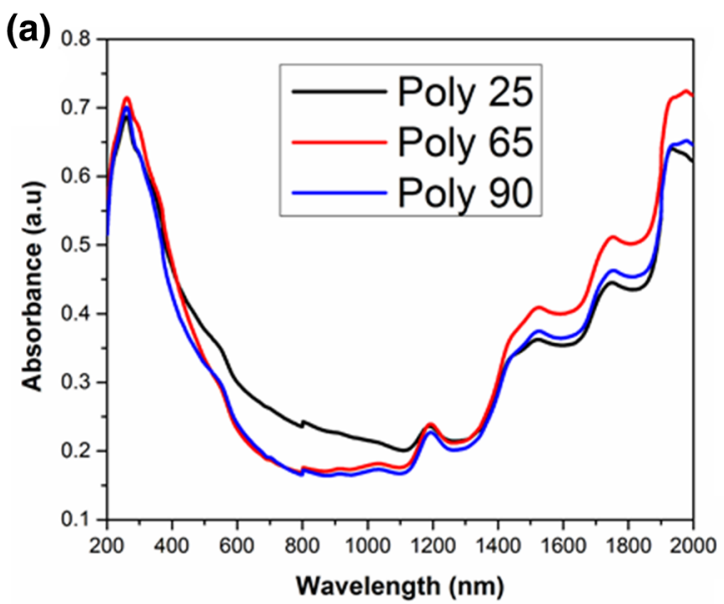

(b)

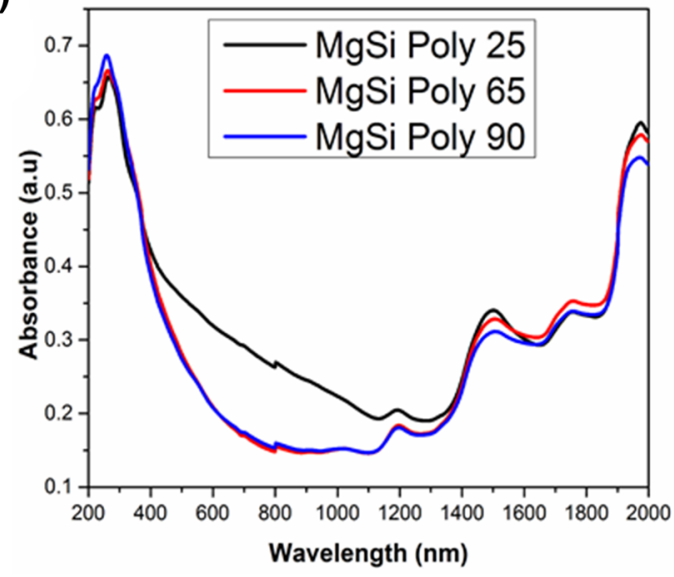

(c)

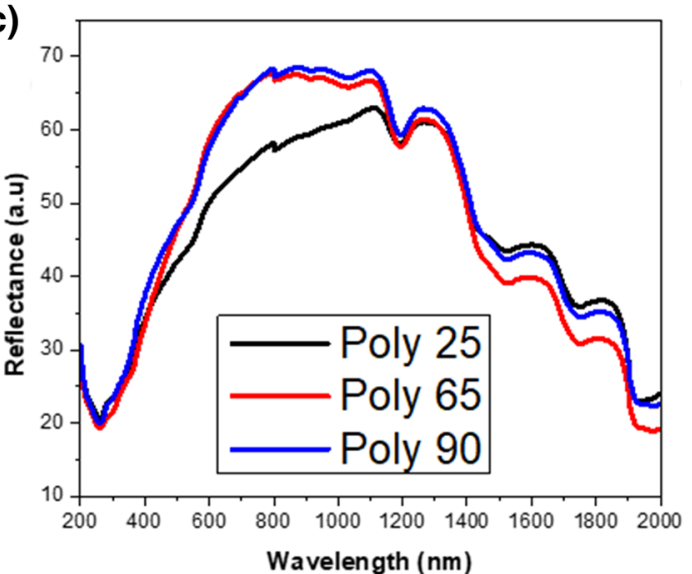

(d)
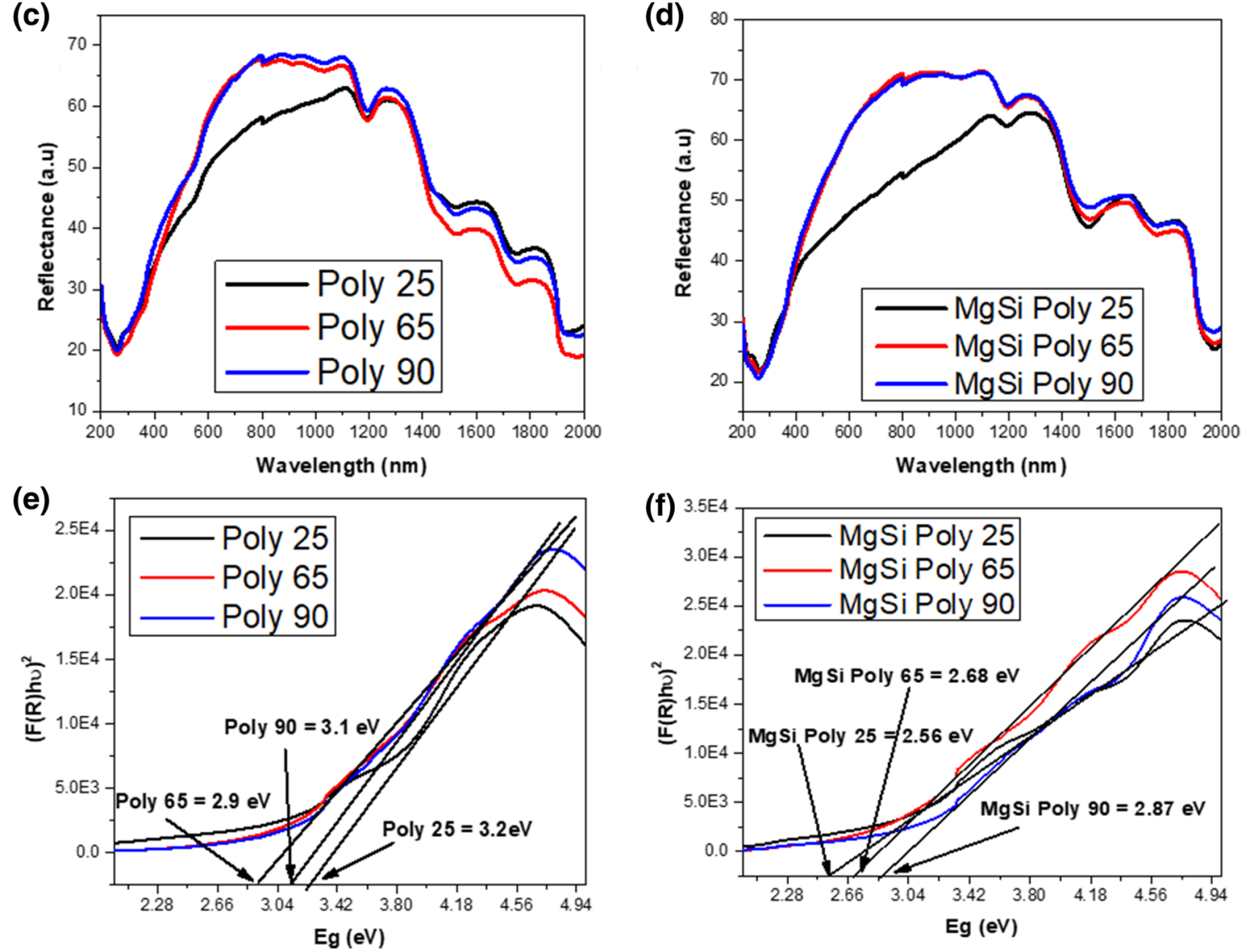

Figure 5. (a) and (b) Absorbance, (c) and (d) diffuse reflectance, (e) and (f) optical band gap of Poly 25, Poly 65 and Poly 90, MgSi Poly 25, MgSi Poly 65 and MgSi Poly 90 samples.

In this specific situation, the differences in the structures for Poly 25, 65 and 90, MgSi Poly 25, 65 and 90 samples were affected by the optical properties of the materials. Figure $5 \mathrm{a}$ and $\mathrm{b}$ shows the absorbance range for Poly 25, 65 and 90, MgSi Poly 25, 65 and 90 samples. The absorption onset of Poly 25, 65 and 90; MgSi Poly
25, 65 and 90 samples was recorded at 385, 430, 400, 470, 465 and $430 \mathrm{~nm}$, respectively (table 2). Accordingly, the radiation measurements at 25 give a viable approach to continuously tune the retention ability of MgSi Poly 25 films covering a large area from 380 to $470 \mathrm{~nm}$. For all samples, they demonstrate the band peaks at 300 and 
Table 2. Optical properties of Poly 25, 65 and 90, MgSi Poly 25, 65 and 90 samples.

\begin{tabular}{lcccc}
\hline Sample & Radiation dose (kGy) & Abs. edge & Optical band gap (eV) & Refractive index \\
\hline Poly 25 & 25 & 385 & 3.2 & 2.345 \\
Poly 65 & 65 & 430 & 2.9 & 2.424 \\
Poly 90 & 90 & 400 & 3.1 & 2.370 \\
MgSi Poly 25 & 25 & 470 & 2.65 & 2.498 \\
MgSi Poly 65 & 65 & 465 & 2.68 & 2.489 \\
MgSi Poly 90 & 90 & 430 & 2.87 & 2.432 \\
\hline
\end{tabular}

$520 \mathrm{~nm}$, because of the metal-to-ligand charge-exchange (MLCT) and ligand-to-metal charge exchange (LMCT) progress which were clearly observed, individually [42].

In turn, the presence of an NIR band between 1100 and $2000 \mathrm{~nm}$ is attributed to the metal-to-metal charge exchange (MMCT) [43]. The structural properties of composite materials have obviously demonstrated that the NIR was high intensity and relies upon the electronic condition of the radiation dosage. Diffuse reflectance UV-Vis spectra for the samples were additionally recorded to determine the optical band gap $\left(E_{\mathrm{g}}\right)$ (figure $5 \mathrm{c}$ and d). The optical band gap energy can be estimated from the UV-Vis spectra in the diffuse reflectance mode. Then, the optical absorption coefficient $(\alpha / S)$ was calculated using the reflectance data according to the Kubelka-Munk equation [36,43,44]. A plot of $(\alpha h v)^{2}$ against $h v$ (figure 5e and f) was linear confirming the direct band gap energy in composite films. Consequently, the calculated band gap energies for Poly 25, 65 and 90, MgSi Poly 25, 65 and 90 samples were found to be 3.2, 2.9, 3.1, $2.65,2.68$ and $2.87 \mathrm{eV}$, respectively. A comparison of these data is presented in table 2. Basically, the optical band gap energy varied with different radiation doses. Obviously, the MgSi Poly 25 was found to have a low band gap energy of $2.65 \mathrm{eV}$ [45]. To analyse the physical properties of the polymers, the calculated values of the refractive index were calculated using the conventional formula. The refractive index shown in table 2 can be calculated by using the following equation [46]:

$$
R=\frac{(n-1)^{2}}{(n+1)^{2}}
$$

The comparison between optical and refractive index properties of Poly 25, 65 and 90, MgSi, MgSi Poly 25, 65 and 90 samples is shown in figure 6 .

\subsection{Dielectric properties}

Frequency and temperature dependence of dielectric constant and dielectric loss of Poly 65 and MgSi 25 samples has been studied in the temperature range $300-600 \mathrm{~K}$ at different frequencies of 100, 500, 1000, 2000 and $4000 \mathrm{~Hz}$. The powder was compressed in a stainless dye under five tones to form the disc. The disc dimensions were about $1 \mathrm{~cm}$ diameter and

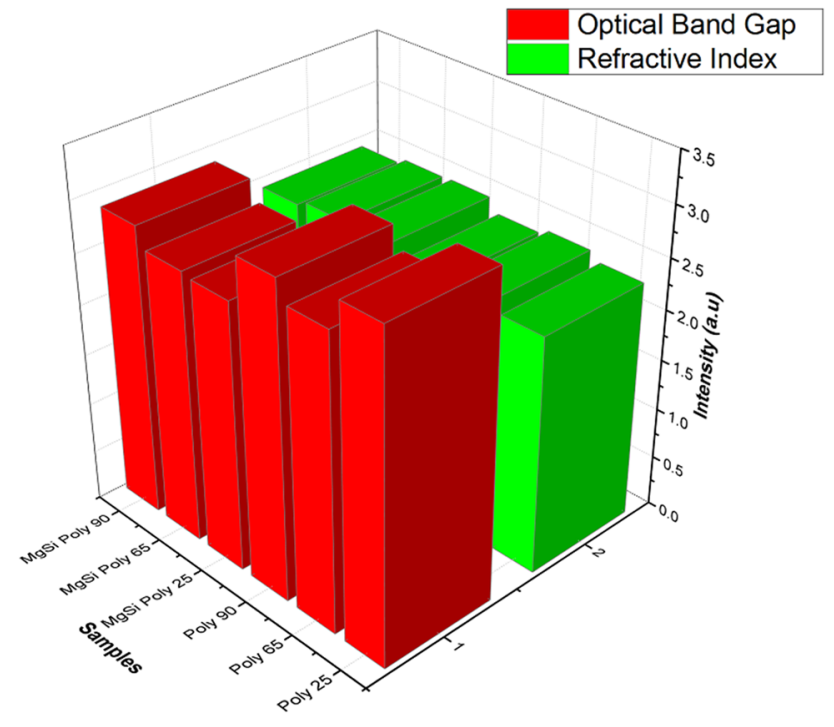

Figure 6. The comparison of optical and refractive index for Poly 25, 65 and 90, MgSi Poly 25, 65 and 90 samples.

$1.5 \mathrm{~mm}$ thickness. Additionally, the two faces of the disc were polished and coated with a thin layer of conducting silver paste to ensure electrical contact. Figure 7 depicts the variation in fixed frequency for the Poly 65 and $\mathrm{MgSi} 25$ samples as a function of temperature from 300 to $\sim 600^{\circ} \mathrm{C}$. The dielectric displacement $(D)$ found in a dielectric material when subjected to an alternating field $(E)$ was given by [47],

$$
D=\varepsilon^{*} \times E
$$

where $\varepsilon^{*}$ is the dielectric constant of the polymer compounds given as follows:

$$
\varepsilon=\varepsilon^{\prime}+j \varepsilon^{\prime \prime}
$$

For an alternating field, the displacement $(D)$ and electric field $(E)$ were not in phase. Hence, the dielectric constant was a complex quantity involving real $\left(\varepsilon^{\prime}\right)$ and imaginary $\left(\varepsilon^{\prime \prime}\right)$ parts, 
(a)

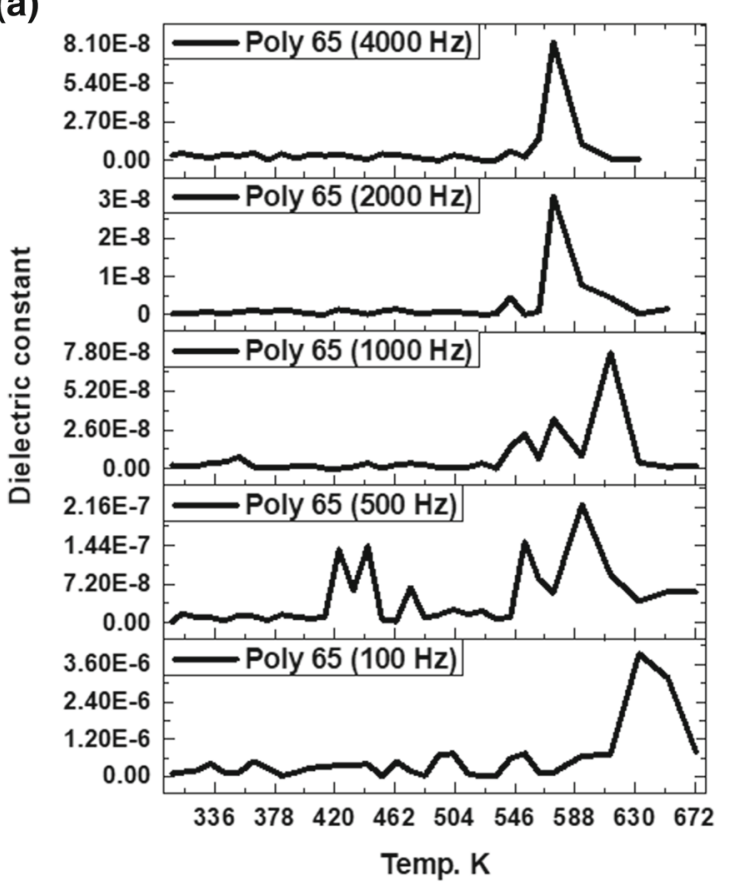

(c)

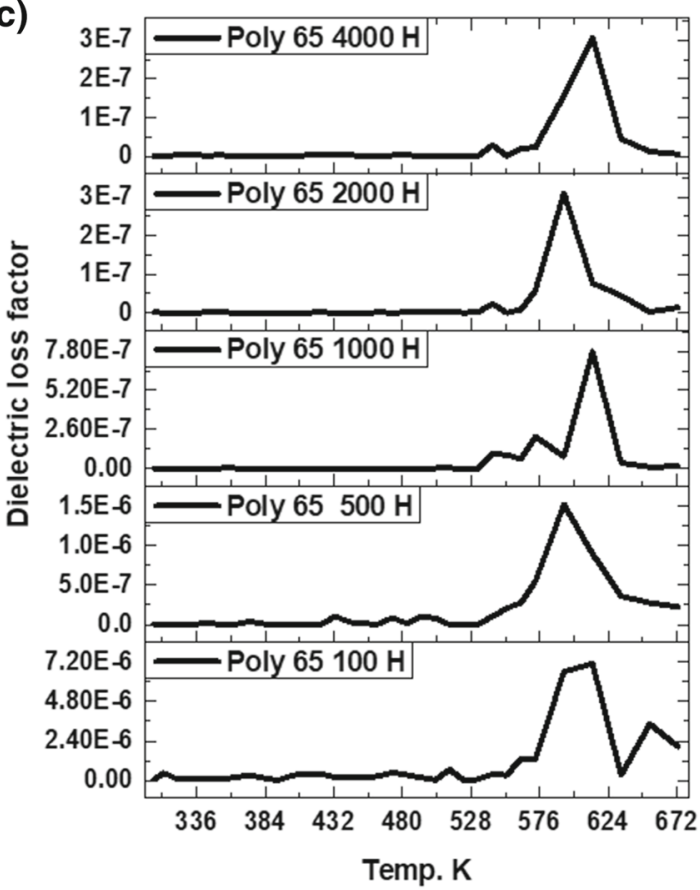

(b)

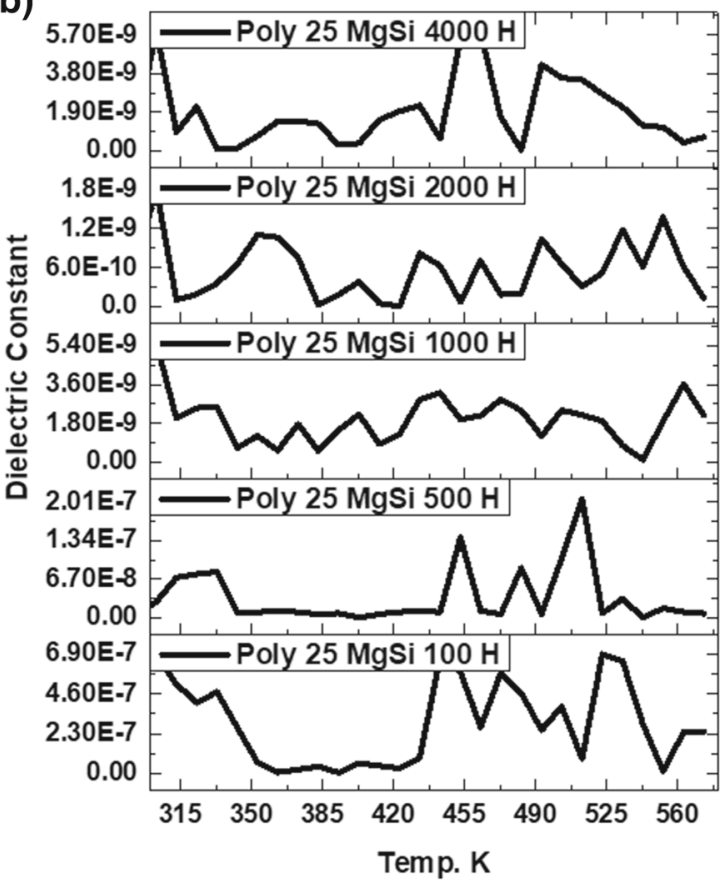

(d)

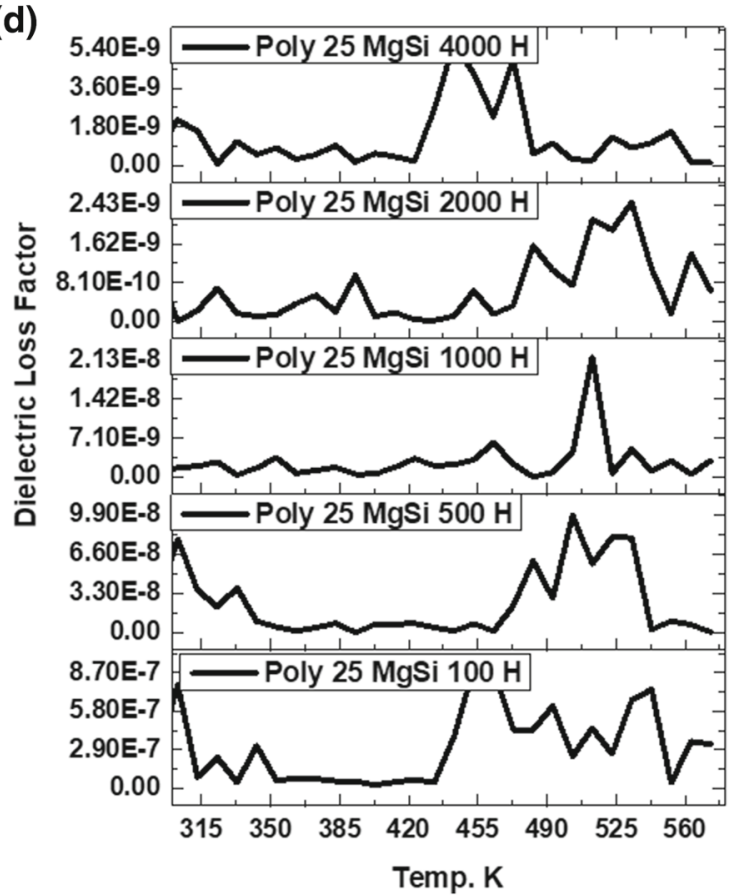

Figure 7. The frequency and temperature dependence of (a) and (b) dielectric constant and (c) and (d) dielectric loss in Poly 65 and MgSi Poly 25 samples.

which were related by a loss factor $(\tan \delta)$ as,

$$
\tan \delta=\frac{\varepsilon^{\prime}}{\varepsilon^{\prime \prime}}
$$

Therefore, the power loss per unit volume of the material is given by

$$
P=\omega E^{2} \varepsilon_{0} \varepsilon^{\prime} \tan \delta
$$

where symbols have their usual meaning. The frequency and time dependence of the complex dielectric constant (assuming Koop's two-layer model) can be given as,

$$
\varepsilon^{*}\left(\frac{\varepsilon_{0}+\varepsilon_{0}-\varepsilon_{0}}{1+\omega^{2} \tau^{2}}\right)+i\left(\frac{\varepsilon_{0}-\varepsilon_{\infty}}{1+\omega^{2} \tau^{2}}\right) \omega \tau
$$


Table 3. Dielectric properties of Poly 25, 65 and 90, MgSi, MgSi Poly 25, 65 and 90 samples.

\begin{tabular}{lcccccc}
\hline & & \multicolumn{2}{c}{ Poly 65} & & \multicolumn{2}{c}{ MgSi Poly 25 } \\
\cline { 3 - 4 } \cline { 6 - 7 } Sample (Hz) & Temp. (K) & Dielectric const. & Dielectric loss & Temp. (K) & Dielectric const. & Dielectric loss \\
\hline 100 & 613 & $3.8 \times 10^{-6}$ & $7.0 \times 10^{-7}$ & 530,453 & $7.1 \times 10^{-7}$ & $9.0 \times 10^{-7}$ \\
500 & 591 & $2.1 \times 10^{-7}$ & $1.5 \times 10^{-6}$ & 513,503 & $2.1 \times 10^{-7}$ & $9.8 \times 10^{-8}$ \\
1000 & 612 & $7.5 \times 10^{-8}$ & $7.7 \times 10^{-7}$ & 563,512 & $3.4 \times 10^{-9}$ & $2.1 \times 10^{-8}$ \\
2000 & 594 & $3.0 \times 10^{-8}$ & $3.0 \times 10^{-7}$ & 553,532 & $1.3 \times 10^{-9}$ & $2.4 \times 10^{-9}$ \\
4000 & 613 & $8.1 \times 10^{-8}$ & $3.0 \times 10^{-7}$ & 463,443 & $5.7 \times 10^{-9}$ & $5.6 \times 10^{-9}$ \\
\hline
\end{tabular}

From equations ( 3 and 4 ), we have $\tan \delta=$ loss current/ charging current, and then the Debye equation is given as follows [48]:

$$
\tan \delta \frac{\left(\varepsilon_{0}-\varepsilon_{\infty}\right) \omega \tau}{\varepsilon_{0}-\varepsilon_{\infty} \omega^{2} \tau^{2}}
$$

The $\varepsilon$ has the highest value and the curve has an inflective point. In this paper, the dielectric properties were demonstrated for the best characteristic samples. From figure 7a, an important observation can be made that the dielectric constant decreases considerably with the increasing of frequency in Poly 65 and MgSi Poly 25 samples. The dielectric constants $(\varepsilon)$ value of Poly 65 and $\mathrm{MgSi}$ Poly 25 at $100 \mathrm{~Hz}$ were $3.8 \times 10^{-6}$ and $7.1 \times 10^{-7}$, which can be explained by the polymer having a higher temperature and thus resulting in higher $\varepsilon$ dielectric constant values. While, the $\varepsilon$ values of Poly 65 and MgSi Poly 25 at $2000 \mathrm{~Hz}$ were $3.0 \times 10^{-8}$ and $1.3 \times 10^{-9}$, respectively. These values were the lowest with increasing the applied frequency as shown in table 3. It is evident from figure $7 \mathrm{a}$ and $\mathrm{b}$ that dielectric constant values decrease with increasing frequency at various temperatures. It is also evident from these figures that decrease was very prominent at both low frequencies and at high temperatures [49]. The decline of $\varepsilon$ with increasing frequency was the normal conduct in most dielectric materials. This was because of dielectric relaxation which was the main reason for a typical dispersion. From a basic perspective, the dielectric relaxation includes the orientation polarization which thus relies on the sub-atomic of dielectric to be material. In this way, at higher frequencies, the rotational movement of the polar particles of the dielectric was not adequately fast for the accomplishment of harmony with the field. Subsequently, the dielectric constant in all samples diminishes with increasing frequency [50,51].

On the other hand, figure $7 \mathrm{c}$ and $\mathrm{d}$ shows the variation of a dielectric loss factor $(\delta)$ with frequency at different temperatures for the Poly 65 and MgSi Poly 25 samples. Generally, the $\tan \delta$ decreases with increasing frequency at different temperatures for the Poly 65 and MgSi Poly 25 samples. However, the composite materials revealed an absorbed dampness at the interface. These special characteristics have increased the mobility of the polymer chain and therefore convey the loss peak because $T_{\mathrm{g}}$ has the epoxy-like shape at high-frequency which has to be identified in our measurement [52]. It can be observed that the dielectric loss factor increased with the temperature, especially at bring down frequency. This resulted in the chain movement of the polymer becoming faster. Also, the glass state has changed the temperature of the polymer. At high frequencies, the dielectric loss was lower and remained consistent with increasing temperature on the grounds. The introduction of polarization because of chain movement of the polymer cannot keep up with the quickly wavering electric field $[53,54]$. This work alluded to the similitude of dielectric phenomena which have seen in different materials. Moreover, these phenomena can be elucidated as being characteristics of materials that have microstructures containing conducting and dielectric phases. The expansion in relative permittivity $\varepsilon^{\prime}$ with the decreasing in estimation frequency was a piece of the 'All-inclusive Dielectric Response' identified by Jonscher [55] as summarized in table 3.

\subsection{Conductivity properties}

The plots of $\ln \sigma_{\text {ac }} v s .1000 / \mathrm{T}$ at different frequencies are shown in figure 8 . The conductivity behaviour for Poly 65 is shown in figure $8 \mathrm{a}$. It was observed that the electrical conductivity has lower values in the range of $34-298^{\circ} \mathrm{C}$. As the temperature increases from 298 to $393^{\circ} \mathrm{C}$, the electrical conductivity sharply increases to a higher value [56]. On the other hand, figure $8 \mathrm{~b}$ reveals the characteristic of the $\mathrm{MgSi} 25 \mathrm{sam}$ ple. It can be seen that the initial conductivity increases up to $87^{\circ} \mathrm{C}$, followed by a moderate increase up to $300^{\circ} \mathrm{C}$, and then a final rapid decrease from 300 to $441^{\circ} \mathrm{C}$. This behaviour was assigned to the conversion of polymer to metallic $\mathrm{MgSi}$, elimination of organic components from the polymer phase to form the composites of magnesium silicate. Narasimha Rao and Subba [57] reported thermally stimulated discharge currents in the composite polymer as a function of the polarizing field strength and concluded that the Poole-Frenkel model describes the dominant mechanism. Blythe [58] stated that polyamides show pronounced ionic conduction effects at elevated temperatures, evidently as a result of the dissociation of amide groups to give protons [59]. In particular, incorporation of metals into the polymer resulted in great improvement in the electrical conductivity of the composites. 

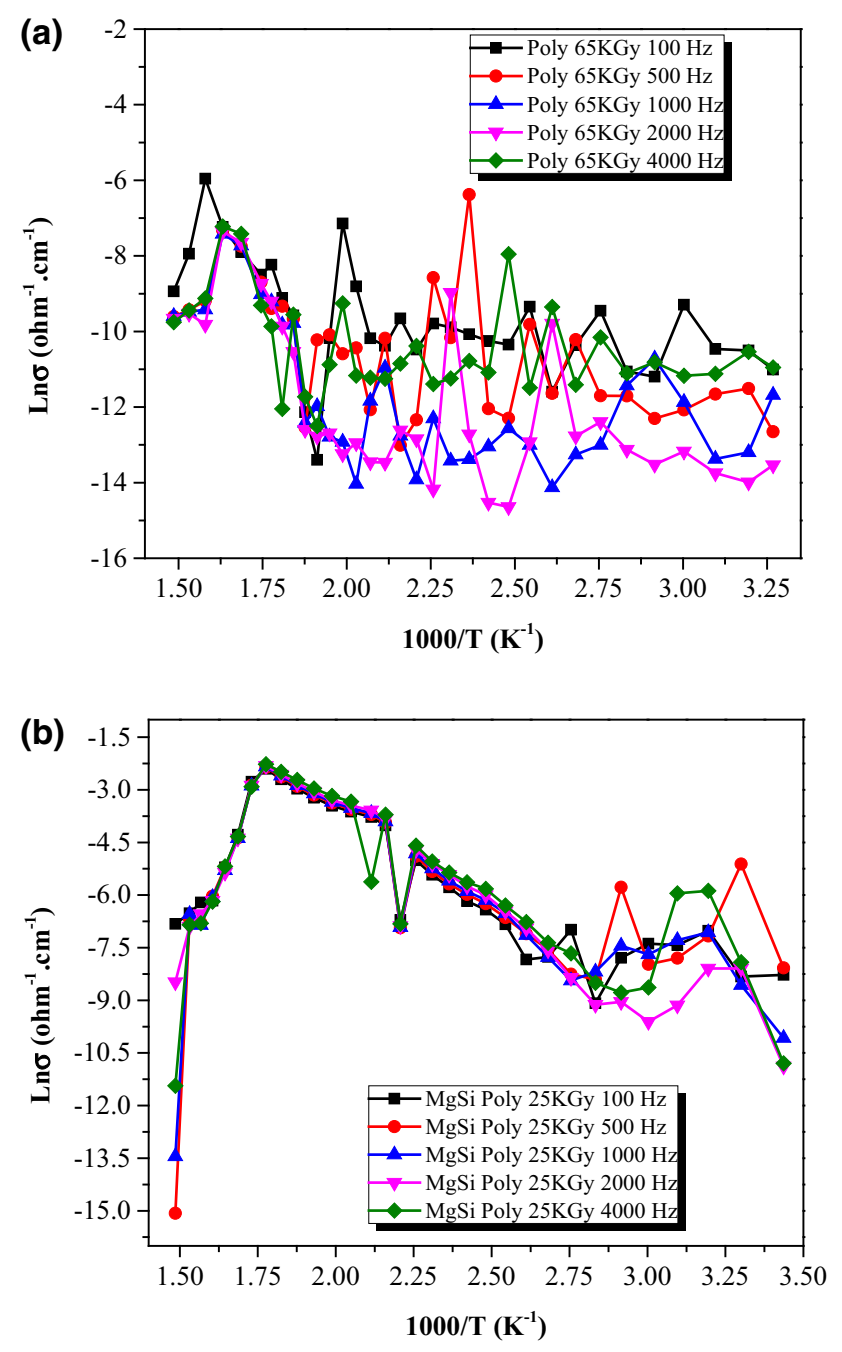

Figure 8. The variation of $\sigma_{\mathrm{ac}}$ vs. $1000 / \mathrm{T}$ of (a) Poly 65 and (b) MgSi Poly 25 samples.

\section{Conclusions}

$\mathrm{MgSi}$ based on polymeric materials have been successfully prepared via $\gamma$-irradiating systems with 25,65 and $90 \mathrm{kGy}$. The XRD patterns uncovered that they had a specific comparability with some distinction identified with radiation measurement. The optical band gap for these materials revealed the photoactive characteristic as a response to light or electromagnetic radiation. The reduction in temperature with the raise in frequency was because of the introduction of polarization and the expansion in the dielectric loss factor. The raise in temperature was because of a tendency for the development of the dipole chain of the polymer at high temperature. Dielectric loss peaks in the composite materials at high temperature were because of the glass progress temperature of the composite polymer. This research could provide a practical guidance in the manufacturing of electrical conductive polymer composites for desirable applications.

\section{Acknowledgements}

The authors would like to extend their sincere appreciation to Central Metallurgical Research and Development Institute, Egypt for its financial support to pursue this work.

\section{References}

[1] Majhi M, Choudhary R and Maji P 2015 Bull. Mater. Sci. 38 1195

[2] Karadă̆ E, Topaç F, Kundakci S H and Üzüm Ö B 2014 Bull. Mater. Sci. 371637

[3] Murali K R and Rao D R 1981 Thin Solid Films 86283

[4] Raghuvanshi S K, Ahmad B, Siddhartha Srivastava A K, Krishna J B M and Wahab M A 2012 Nucl. Instrum. Methods Phys. Res. Sect. B Beam Interact. Mater. At. 27144

[5] Burland D M, Miller R D and Walsh C A 1994 Chem. Rev. 94 31

[6] Angiolini L, Benelli T, Giorgini L and Salatelli E 2006 Polymer 471875

[7] Rosseinsky D R and Mortimer R J 2001 Adv. Mater. 13783

[8] Grote J G, Zetts J S, Nelson R L, Hopkins F K, Dalton L R, Zhang C et al 2001 Opt. Eng. 402464

[9] Liu C-L and Chen W-C 2011 Polym. Chem. 22169

[10] Katz E and Willner I 2003 J. Am. Chem. Soc. 1256803

[11] Özdemir T, Güngör A, Akbay I K, Uzun H and Babucçuoglu Y 2017 Radiat. Phys. Chem. 144248

[12] Alekseev V, Baranovsky V, Vedenov A, Velichko A, Zaytzeva L, Kovalenko A et al 1991 Bull. Mater. Sci. 14257

[13] Lawton E, Bueche A and Balwit J 1953 Nature 17276

[14] Ambika M R, Nagaiah N, Harish V, Lokanath N K, Sridhar M A, Renukappa N M 2017 Radiat. Phys. Chem. 130351

[15] Sharma T, Aggarwal S, Kumar S, Mittal V K, Kalsi P C and Manchanda V K 2007 J. Mater. Sci. 421127

[16] Moez A A, Aly S S and Elshaer Y H 2012 Spectrochim. Acta Part A Mol. Biomol. Spectrosc. 93203

[17] Nagarale R K, Shin W and Singh P K 2010 Polym. Chem. 1 388

[18] Armstrong B L, Campbell A A, Gutowska A and Song L 2002 Polymer/ceramic composites (Google Patents)

[19] Rao Y and Wong C 2004 J. Appl. Polym. Sci. 922228

[20] Rao Y, Ogitani S, Kohl P and Wong C 2002 J. Appl. Polym. Sci. 831084

[21] Newnham R E 1986 Ferroelectrics 681

[22] Kuo D-H, Chang C-C, Su T-Y, Wang W-K and Lin B-Y 2001 J. Eur. Ceram. Soc. 211171

[23] Qingbo Z, Liying Z and Yuzhen S 1993 Radiat. Phys. Chem. 4273

[24] Hassan M F and Yusof S Z M 2014 Microsc. Res. 230

[25] Kangwansupamonkon W, Jitbunpot W and Kiatkamjornwong S 2010 Polym. Degrad. Stab. 951894

[26] Hassan A M, Zakaria E S, Ibrahim A B, Abass M R and AbouMesalam M M 2018 Int. J. Innov. Res. Growth 666

[27] Manuel Stephan A and Nahm K S 2006 Polymer 475952

[28] Zhang T, Vandeperre L J and Cheeseman C R 2014 Cem. Concr. Res. 658

[29] Kato T, Kinoshita Y, Nishiyama N, Wada K, Zhou C and Irifune T 2014 Phys. Earth Planet. Inter. 23226

[30] Abdel-Galil E, El-Deen G S, El-Aryan Y and Khalil M 2016 Russ. J. Appl. Chem. 89467 
[31] Li D, Wang M, Yang C, Wang J and Ren G 2012 Chem. Pharm. Bull. 60995

[32] Deng Y, Dixon J B, White G N, Loeppert R H and Juo A S 2006 Colloids Surf. A Physicochem. Eng. Asp. 28182

[33] Fournier J A, Johnson C J, Wolke C T, Weddle G H, Wolk A B and Johnson M A 2014 Science 3441009

[34] Rashidzadeh A, Olad A, Salari D and Reyhanitabar A 2014 J. Polym. Res. 21344

[35] Xiao-Hong L, Hong-Ling C, Rui-Zhou Z and Xian-Zhou Z 2015 Spectrochim. Acta Part A Mol. Biomol. Spectrosc. 137 321

[36] Rashad M M, Elseman A M and Hassan A M 2016 Optik-Int. J. Light Electron Opt. 1279775

[37] Luo B, Wang X, Wang Y and Li L 2014 J. Mater. Chem. A 2 510

[38] Shalabney A, George J, Hutchison J, Pupillo G, Genet C and Ebbesen T W 2015 Nat. Commun. 65981

[39] Abou-Mesalam M M, Abass M R, Abdel-Wahab M A, Zakaria ES, Hassan A M and Khalil HF 2016 Desalination Water Treat. 5725757

[40] Madejová J 2003 Vib. Spectrosc. 311

[41] Anwar A, Elfiky D, Ramadan A M and Hassan G M 2017 Radiat. Phys. Chem. 13414

[42] Maryanski M, Zastavker Y and Gore J 1996 Phys. Med. Biol. 412705

[43] Elseman A, Shalan A, Rashad M and Hassan A 2017 Mater. Sci. Semicond. Process. 66176
[44] Elseman A M, Shalan A E, Rashad M M, Hassan A M, Ibrahim N M and Nassar A M 2017 J. Phys. Org. Chem. 30 e3639

[45] Rashad M, Hassan A, Nassar A, Ibrahim N and Mourtada A 2014 Appl. Phys. A 117877

[46] Thayer G D 1974 Radio Sci. 9803

[47] Chia L H L, Chua P H, Hon Y S and Lee E 1986 Int. J. Radiat. Appl. Instrum. Part C Radiat. Phys. Chem. 27207

[48] Jonscher A K 1977 Nature 267673

[49] Aras L and Baysal B M 1984 J. Polym. Sci. Part B Polym. Phys. 221453

[50] Andreuccetti D, Bini M, Ignesti A, Olmi R, Rubino N and Vanni R A 1988 IEEE Trans. Biomed. Eng. 35275

[51] Pant H, Patra M, Negi S, Bhatia A, Vadera S and Kumar N 2006 Bull. Mater. Sci. 29379

[52] Song H-S, Yang C and Liu D-B 2012 J. Funct. Mater. 9 25

[53] Zikry A 2008 Int. J. Polym. Mater. 57383

[54] Manouras T and Vamvakaki M 2017 Polym. Chem. 874

[55] Jonscher A K 1999 J. Phys. D: Appl. Phys. 32 R57

[56] Nassar A M, Abo Zeid E F, Elseman A M and Alotaibi N F 2018 New J. Chem. 421387

[57] Rao V and Rao B S 1991 Acta Polym. 42379

[58] Blythe A R and Bloor D 2005 Electrical properties of polymers (United Kingdom: University Press)

[59] Maji P, Pande P and Choudhary R 2015 Bull. Mater. Sci. 38 417 